\title{
Up-regulation of DRAM2 promotes tolerance of bladder transitional cell carcinoma to gemcitabine
}

\author{
Baihetiya Azhati, Naibijiang Maolakuerban, Tao Ma, Xiaodong Li, Mulati Rexiati
}

Department of Urology, First Affiliated Hospital of Xinjiang Medical University, Urumchi, Xinjiang, China

Submitted: 29 September 2017

Accepted: 18 December 2017

Arch Med Sci 2020; 16 (5): 1207-1217

DOI: https://doi.org/10.5114/aoms.2020.93748

Copyright $\odot 2020$ Termedia \& Banach

\section{Abstract}

Introduction: Bladder transitional cell carcinoma (BTCC) is one of the most prevalent human malignant diseases. Gemcitabine is commonly applied in the treatment of BTCC while acquired gemcitabine resistance has caused a severe impediment to recovery. This study aimed to investigate the function of DRAM 2 in regulating gemcitabine resistance of BTCC.

Material and methods: GSE77883 was introduced to screen out the differentially expressed autophagy-related genes in T24 cells and gemcitabineresistant T24-GEM cells. After establishing T24-GEM cells ourselves, aberrant expression of DRAM 2 was detected by qRT-PCR and Western blot. After stably manipulating the expression of DRAM2 in T24 and T24-GEM cells, the changes of cell biological functions under gemcitabine treatment were compared, including cell viability, apoptosis and autophagy, using colony formation, flow cytometry and electron microscopy respectively.

Results: DRAM2 was up-regulated in gemcitabine-resistant T24-GEM cells. Silencing of DRAM2 in T24-GEM cells inhibited the cell autophagy induced by treatment with gemcitabine and contributed to attenuated gemcitabine resistance. Also, overexpression of DRAM2 in T24 cells enhanced the autophagy, strengthened the chemoresistance and decreased the cell apoptosis rate under the treatment with gemcitabine.

Conclusions: Our data suggested that downregulation of DRAM2 rescued the sensitivity of T24-GEM cells to gemcitabine, providing an appropriate therapeutic target for BTCC treatment.

Key words: bladder cancer, DRAM2, autophagy, gemcitabine.

\section{Introduction}

Bladder cancer, as one of the most prevalent genitourinary malignancies, is characterized by its high recurrence and mortality rate [1]. Transitional cell carcinoma (TCC) represented $90 \%$ of all the bladder cancer cases diagnosed in the USA [2]. Although some achievements in chemotherapy regimens improved the therapies of bladder cancer, the 5-year survival rate of bladder transitional cell carcinoma (BTCC) patients still ranges from 48 to $66 \%$ [3]. Therefore, the development of new therapies is still desirable. Gemcitabine, a nucleoside antimetabolite that inhibits DNA synthesis, is one of the most often used anticancer drugs in the treatment of BTCC $[4,5]$. However, the development of chemoresistance for gemcitabine has become one of the most important reasons for treatment failure and has resulted in relapse, metastasis or patient death [6]. In order to improve

\author{
Corresponding author: \\ Mulati Rexiati \\ Department of Urology \\ First Affiliated Hospital \\ of Xinjiang Medical University \\ 393 Xinyi Road \\ Xinshi District \\ Urumchi, 830054 \\ Xinjiang, China \\ Phone: +86 0991-4362718 \\ E-mail: suiyanf@163.com
}


the therapeutic efficacy of BTCC, it is urgent to advance our understanding of mechanisms for gemcitabine-induced resistance and increase tumor cell sensitivity to gemcitabine [7].

The gene DRAM2 (DNA-damage-regulated autophagy modulator protein 2), also known as TMEM77 (transmembrane protein 77), encodes a 266-amino acid protein with six putative transmembrane domains [8]. Localized to lysosomal membranes, $D R A M 2$ plays a role in autophagy induction via promoting the conversion of endogenous LC3-I (microtubule-associated protein light chain 3) to the general autophagosome marker protein LC3-II (LC3-I/ phosphatidylethanolamine conjugate) [9]. LC3 is required for the elongation of autophagosomes, which has two forms: LC3-I and LC3-II [10]. LC3-II, as the most reliable marker for quantification of cell autophagy, is up-regulated when LC3-I converts to LC3-II during autophagy [10].

Autophagy is a highly conserved process. The function of autophagy is to sequester parts of the cytoplasm, including damaged, superfluous organelles or long-lived proteins, into autophagosomes, which are double-membrane vesicles [7]. Autophagy serves an essential role in maintaining tissue homeostasis to support cell growth and survival [4], such as inflammatory bowel disease, neuronal degeneration, aging and cancer. On the other hand, many studies have reported that autophagy is a significant mechanism in chemoresistance, and inhibition of autophagy may enhance the sensitivity of cancer cells to chemotherapy [11], such as breast cancer, non-small cell lung cancer cells [12] and colorectal cancers [13].

Given these findings, we hypothesized that DRAM 2 mediates chemoresistance in bladder cancer cells. Therefore, we set out to test this hypothesis by investigating the relationship between DRAM2 and autophagy in gemcitabine sensitive/ resistance BTCC cells. The results determined the relationship between expression of DRAM2 and autophagy, suggesting a promising new combination in the treatment of bladder transitional cell carcinoma.

\section{Material and methods}

\section{Integrated analysis of microarray datasets}

The microarray data in the GEO database (accession number GSE77883) at the National Center for Biotechnology Information Gene Expression Omnibus (http://www.ncbi.nlm.nih.gov/geo/) under GPL19117 were used to analyze the six samples (three T24 cells and three T24-GEM cells). Differentially expressed mRNAs related to autophagy were identified based on the criteria of over 2-fold expression change within different groups and a $p$-value less than 0.05 .

\section{Cell culture}

Bladder transitional cell carcinoma cell lines T24 (TCHu 55) were purchased from the Cell Bank of the Chinese Academy of Sciences (Shanghai, China). Cells were cultured in RPMI 1640 (Roswell Park Memorial Institute, Thermo Fisher Scientific, Waltham, MA, USA), supplemented with $10 \%$ fetal bovine serum (FBS), $100 \mathrm{U} / \mathrm{ml}$ penicillin, and $100 \mathrm{ug} / \mathrm{ml}$ streptomycin. Gemcitabine-resistant BTCC cells (T24-GEM) were derived by treating T24 cells with $5 \mu \mathrm{g} / \mathrm{ml}$ gemcitabine and maintained in the same condition for more than 6 months. The T24-GEM cells were cultured in complete medium supplemented with $12 \%$ FBS, $5 \mu \mathrm{g} / \mathrm{ml}$ gemcitabine, $100 \mathrm{U} / \mathrm{ml}$ streptomycin and $100 \mathrm{U} / \mathrm{ml}$ penicillin. Cells were incubated at $37^{\circ} \mathrm{C}$ with $5 \%$ $\mathrm{CO}_{2}$ in an incubator.

\section{Real-time PCR analysis}

Total RNA was prepared from T24/T24-GEM cells transfected with DRAM2/si-DRAM2 according to the manufacturer's instructions and the concentration of total RNA was determined. The RNA was then reverse transcribed in a $20 \mu \mathrm{l}$ reaction mixture according to the instructions of the RevertAid First strand cDNA syntherio Kit (Fermentas, China). The PCR program for DRAM2 and GAPDH (Santa Cruz Biotechnology, CA, USA) included one cycle at $95^{\circ} \mathrm{C}$ for $2 \mathrm{~min}, 39$ cycles at $95^{\circ} \mathrm{C}$ for $20 \mathrm{~s}, 65^{\circ} \mathrm{C}$ for $20 \mathrm{~s}, 72^{\circ} \mathrm{C}$ for $30 \mathrm{~s}$, and finally extension at $72^{\circ} \mathrm{C}$ for $10 \mathrm{~min}$. The PCR products of the DRAM 2 gene and internal controls were detected by electrophoresis in agarose gel. All DNA fragments and resulting clones were verified by sequencing.

\section{Western blot}

Cells and tissues were lysed with ice-cold RIPA buffer (Beyotime, Shanghai, China) supplemented with phenylmethanesulfonyl fluoride (PMSF) and protease inhibitor cocktail (Roche). Cell protein lysates were subjected in $10 \%$ sodium dodecyl sulfate-polyacrylamide gels, electrophoretically transferred to polyvinylidene difluoride membranes (Roche). Total protein concentration was measured using a BCA protein assay kit before loading for SDS-PAGE. The membranes were incubated with 5\% skim milk, washed and then incubated with the rabbit anti-human DRAM2 (1: 500 dilution) or GAPDH (1: 1000 dilution) overnight at $4^{\circ} \mathrm{C}$, followed by incubated with goat anti-human horseradish peroxidase antibody for $1 \mathrm{~h}$ at $37^{\circ} \mathrm{C}$. All antibodies were purchased from Abcam (Abcam, USA). The proteins were perceived by the enhanced chemiluminescence kit (Invitrogen) and exposed to X-ray film. Protein expression levels were normalized to GAPDH. 


\section{Cell transfection}

Human DRAM2 cDNA and si-DRAM2 were chemically synthesized by Sangon Biotech Co., Ltd. (Shanghai, China). The oligonucleotides were transfected using Lipofectamine 2000 (Invitrogen, Carlsbad, (A, USA) following the manufacturer's protocol. Cells were subcultured 1 day before the transfection and were inoculated into 6-well plates. After cell confluence reached 90-95\%, $50 \mu \mathrm{l}$ of serum-free medium was used to dilute $1.0 \mu \mathrm{g}$ of DNA, and then another $50 \mu \mathrm{l}$ of serumfree medium was used to dilute $1 \mu \mathrm{l}$ of Lipofectamine 3000 transfection reagent. Subsequently, the solution was mixed with the diluted DNA at room temperature for $20 \mathrm{~min}$ and added to each well. Cells were generally assigned to different groups as follows: (1) NC group (transfected with Lipofectamine 2000), siRNA control group and si-DRAM2 group (T24-GEM cells); (2) NC group (transfected with Lipofectamine 2000), pcDNA3.1 control group, pcDNA3.1-DRAM2 group (T24 cells). The transfection efficiency was observed under qRT-PCR and western blot, and siRNA sequences are listed in Table I.

\section{MTT assay}

Cell viability was assessed by using 3-(4,5-dimethylthiazol-2-yl)-2, 5-diphenyltetrazolium bromide (MTT) assay. 3000 cells in $100 \mu$ of medium per well were seeded in 96-well plates. Cells were cultured for $24 \mathrm{~h}, 48 \mathrm{~h}, 72 \mathrm{~h}, 96 \mathrm{~h}$, and then incubated with $0.5 \mathrm{mg} / \mathrm{ml}$ of MTT at $37^{\circ} \mathrm{C}$ for $4 \mathrm{~h}$. Medium was replaced with $150 \mu \mathrm{l}$ DMSO per well to dissolve the precipitates. Colorimetric analysis using a 96-well micro-plate reader (Bio Tek) was performed at wavelength of $490 \mathrm{~nm}$. An effect curve was drawn and the IC50 value of gemcitabine to bladder cancer cells was calculated depending on cell proliferation and drug concentration.

\section{Transmission electron microscopy}

Cells were treated with BIX-01294 $1 \mu \mathrm{M}$ and then fixed in Karnovsky's fixative (2\% paraformaldehyde and $5 \%$ glutaraldehyde in $0.1 \mathrm{M}$ cacodylate, $\mathrm{pH}$ 7.4) followed by osmium tetroxide. Samples were then dehydrated in ethanol, infiltrated and embedded with TAAB Low Viscosity Resin (TLV) mixture at $60^{\circ} \mathrm{C}$ for $24 \mathrm{~h}$ and sectioned to $80 \mathrm{~nm}$ in thickness on 300 mesh copper slot grids. Analysis was performed by transmission electron microscopy (JEOL, JEM-1400) at $60 \mathrm{kV}$.

\section{Apoptosis analysis}

Apoptotic cells were stained using Annexin V-FITC/Propidium lodide (PI) contained in the Apop-
Table I. Primers used in the study

\begin{tabular}{|lc|}
\hline Gene & \multicolumn{1}{c|}{ Sequences (5'-3') } \\
\hline q-DRAM2-F & GTTATCTGGTGTGGAGTAAGT \\
\hline q-DRAM2-R & GTAGCCGTGTTCGTTCAT \\
\hline q-GAPDH-F & ACCCCGCCGCCTGTGGAGG \\
\hline q-GAPDH-R & TTCTGACGGCAGGTCAGGT \\
\hline Si-DRAM2-F & GCAATGCTAAATATTGCGG \\
\hline Si-DRAM2-R & CCGCAATATTTAGCATTGC \\
\hline Si-Control-F & CCTTCGATATAATTATTGC \\
\hline Si-Control-R & GCAATAATTATATCGAAGG \\
\hline cDNA-DRAM2-F & CAACCTGACGTGCACCAATC \\
\hline cDNA-DRAM2-R & CCTACAGGTGCCTCTCTCCT \\
\hline$q-$ used in qRT-PCR, Si - siRNA, F - forward primer, $R-$ reverse
\end{tabular}
primer.

tosis Kit (KeyGene Biotech, Nanjing, China). After $48 \mathrm{~h}$ of transfection, T24/T24-GEM cells were stained with $5 \mu \mathrm{l}$ Annexin V-FITC and $5 \mu \mathrm{l}$ of PI and cultured in the dark at room temperature for $15 \mathrm{~min}$. These cells were analyzed by flow cytometry, and results were processed with CellQuest Pro software (BD Biosciences, San Jose, CA, USA).

\section{Statistical analysis}

Statistical analyses were performed using GraphPad Prism software v. 6.0 (GraphPad Prism software Inc., La Jolla, CA, USA). Data are presented as mean \pm SEM (standard error of mean). All experiments were performed in triplicate and repeated at least three times. The unpaired Student's $t$-test was used for comparison of two groups, and one-way ANOVA was performed to analyze differences among more than two experimental groups. Statistical significance was labeled as: ${ }^{*} p<0.05,{ }^{* *} p<0.01,{ }^{* *} p<0.001$.

\section{Results}

\section{DRAM2 was up-regulated in gemcitabine- resistant cells}

Differentially expressed genes in six cell lines are listed in Table II, DRAM2 highly expressed in T24-GEM cells and lowly expressed in T24 cells. The $P$-value was computed using the $t$-test, and fold changes of gene expression were calculated by the ratio of the average expression level between drug-resistant and drug-sensitive groups (Table III). The fold change value of DRAM2 between T24 cells and T24-GEM cells was $2^{-0.9224}$, and the $p$-value was less than 0.05 . Information of related genes in the heatmap, including full name, position and ID, is shown in Table IV. Five genes were highly expressed in the T24 cells and lowly 
Table II. Relative expression values of differential genes

\begin{tabular}{|lcccccc|}
\hline Gene & $\begin{array}{c}\text { T24_gemcita- } \\
\text { bine.resistant_1 }\end{array}$ & $\begin{array}{c}\text { T24_gemcita- } \\
\text { bine.resistant_2 }\end{array}$ & $\begin{array}{c}\text { T24_gemcita- } \\
\text { bine.resistant_3 }\end{array}$ & T24_1 & T24_2 & T24_3 \\
\hline ATG9B & -0.58597 & -0.28833 & -0.58597 & 0.169592 & 0.431298 & 0.64276 \\
\hline ATG12 & -0.39833 & -0.39833 & -0.39833 & 0.291868 & 0.054718 & 0.984777 \\
\hline ATG4D & -0.21256 & 0.169592 & -0.11866 & 0.984777 & 0.984777 & 0.054718 \\
\hline ATG13 & -0.11866 & -0.25988 & -0.28833 & 0.64276 & 0.506704 & 0.291868 \\
\hline ATG16L1 & -0.28833 & -0.21256 & -0.06436 & 0.506704 & 0.169592 & 0.343298 \\
\hline ATG7 & 0.431298 & 0.64276 & 0.506704 & -0.28833 & -0.21256 & -0.39833 \\
\hline DRAM1 & 0.984777 & 0.343298 & 0.431298 & -0.11866 & -0.39833 & -0.25988 \\
\hline DRAM2 & 0.64276 & 0.506704 & 0.64276 & -0.39833 & -0.28833 & -0.28833 \\
\hline ATG10 & 0.506704 & 0.984777 & 0.984777 & -0.58597 & -0.58597 & -0.58597 \\
\hline
\end{tabular}

Negative values (-) denote low expression and positive ones denote high expression.

Table III. Results of fold-change and $p$-value of differential genes

\begin{tabular}{|c|c|c|c|}
\hline Gene symbol & $\log F C$ & $P$-value & adj. $P$-value \\
\hline ATG9B & -0.901304 & 0.000252951 & 0.001011803 \\
\hline ATG 12 & -0.842114 & 0.001995655 & 0.004561498 \\
\hline ATG4D & -0.728634 & 0.009445382 & 0.01679179 \\
\hline ATG13 & -0.702731 & 0.001070063 & 0.002853502 \\
\hline ATG $16 L 1$ & -0.528279 & 0.00794332 & 0.01588664 \\
\hline ATG7 & 0.82666 & 0.000201318 & 0.001011803 \\
\hline DRAM 1 & 0.84541 & 0.000836573 & 0.002677033 \\
\hline DRAM2 & 0.9224 & $6.02 \mathrm{E}-05$ & 0.000481658 \\
\hline ATG 10 & 1.41139 & $2.45 \mathrm{E}-06$ & $3.91 \mathrm{E}-05$ \\
\hline
\end{tabular}

Negative values (-) denote low expression and positive denote high expression. LogFC represents the gene expression fold change of T24 cells compared with T24 gemcitabine resistant cells. P-value and adj. p-value refer to statistical values, $p<0.05$ indicates statistical significance.

Table IV. Detailed information of the differential genes

\begin{tabular}{|lcccc|}
\hline Genes & \multicolumn{1}{c}{ Full name } & Position & ID \\
\hline ATG9B & ATG9 autophagy related 9 homolog B & chr7:150720259-150720200 & NM_173681 \\
\hline ATG12 & ATG12 autophagy related 12 homolog & chr5:115167432-115167373 & NM_004707 \\
\hline ATG4D & ATG4 autophagy related 4 homolog D & chr19:10663725-10663784 & NM_032885 \\
\hline ATG13 & ATG13 autophagy related 13 homolog & chr11:46690061-46690120 & NM_001205119 \\
\hline ATG16L1 & ATG16 autophagy related 16-like 1 & chr2:234202948-234203007 & NM_030803 \\
\hline ATG7 & ATG7 autophagy related 7 homolog & chr3:11468321-11468380 & NM_006395 \\
\hline DRAM1 & DNA-damage regulated autophagy modulator 1 & chr12:102317112-102317171 & NM_018370.2 \\
\hline DRAM2 & DNA-damage regulated autophagy modulator 2 & chr1:111662564-111662505 & NM_178454.5 \\
\hline ATG10 & ATG10 autophagy related 10 homolog & chr5:81550614-81550673 & NM_001131028 \\
\hline
\end{tabular}

expressed in T24-GEM cells, and four genes were lowly expressed in T24 cells and highly expressed in T24-GEM cells, including DRAM2 (Figure $1 \mathrm{~A}$, $p<0.05)$. Due to three other genes being expressed both in humans and yeast, the DRAM2 gene was selected as the target gene for further 
study. The IC50 value of T24-GEM cells $(9.953 \mu \mathrm{g} /$ $\mathrm{ml}$ ) was significantly higher than that of T24 cells $(2.366 \mu \mathrm{g} / \mathrm{ml})$, and the cell viability of T24-GEM cells was significantly higher than that of T24 cells after treatment with gemcitabine (Figure $1 \mathrm{~B}$, $p<0.01)$. Also, protein expression of DRAM2 in T24-GEM cells was higher than that of T24 cells, which was consistent with the results of microarray analyses (Figure $1 \mathrm{C}$ ).

\section{Silencing of DRAM2 increased the sensitivity of T24-GEM cells to gemcitabine and inhibited autophagy}

To determine whether DRAM2 plays a role in the regulation of cellular autophagy and apoptosis in response to gemcitabine, siRNA against DRAM2 and control siRNA were transfected into T24-GEM cells. We observed a decrease in the expression of mRNA and protein after knockdown of DRAM 2 (Figure $2 \mathrm{~A}, \mathrm{~B}, p<0.05$ ). In the si-DRAM 2 group, T24-GEM cells became more sensitive to gemcitabine-induced damage with the IC50 level decreasing from $9.953 \mu \mathrm{g} / \mathrm{ml}$ to $2.266 \mu \mathrm{g} / \mathrm{ml}$ (Figure 2 C, $p<0.01$ ). We also investigated the viability and apoptosis of these cells using MTT and flow cytometry. The results showed that under the treatment of $0.5 \mu \mathrm{g} / \mathrm{ml}$ gemcitabine, DRAM2-knockdown cells were less active compared with si-Con cells (Figure $2 \mathrm{D}, p<0.01$ ), and the number of apoptotic cells increased (Figure $2 \mathrm{E}$, $p<0.01)$. Also, the number of autophagosome decreased (Figure 2 F). LC3 I level increased while LC3 II level decreased (Figure $2 \mathrm{G}$ ). These results suggested that silencing of DRAM2 decreased T24-GEM cells' sensitivity to gemcitabine and inhibited autophagy.

\section{Over-expression of DRAM2 decreased the sensitivity of T24 cells to gemcitabine and promoted autophagy}

Plasmids of pcDNA 3.1 and pcDNA 3.1-DRAM2 were transfected into T24 cells. Over-expression
A

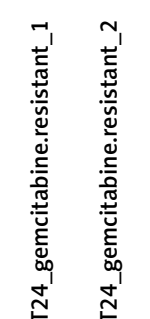
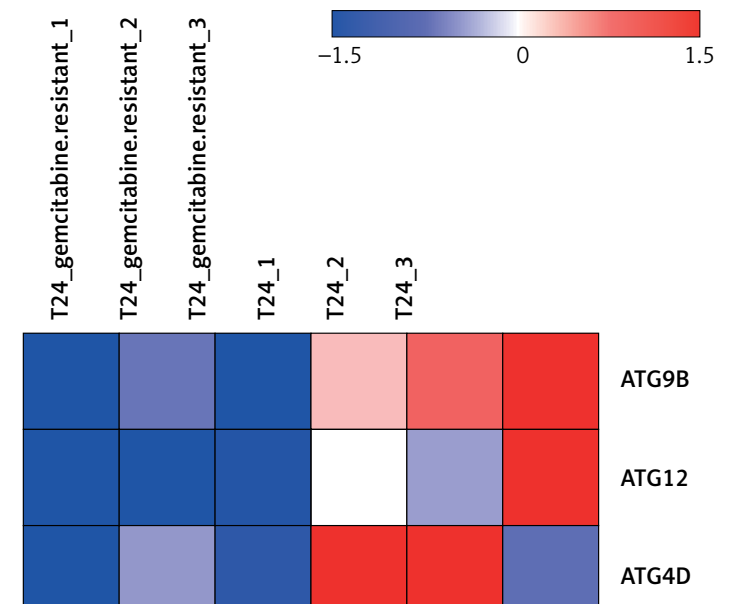

ATG4D

ATG13

ATG16L1

ATG7

DRAM1

DRAM2

ATG10

B

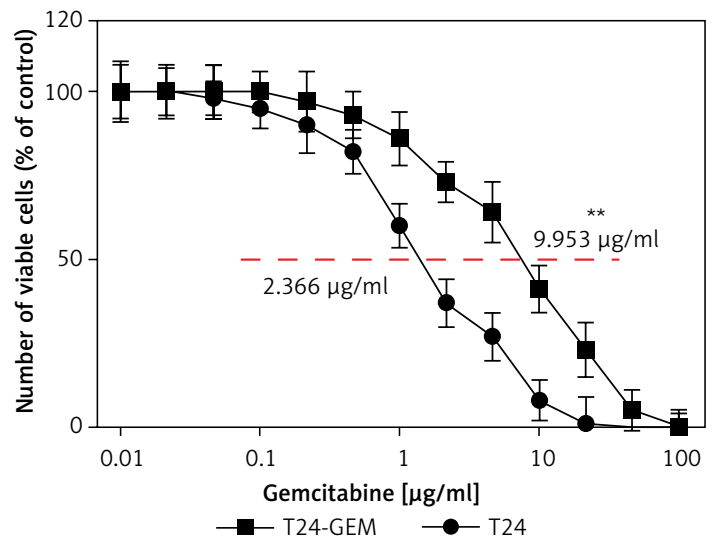

C

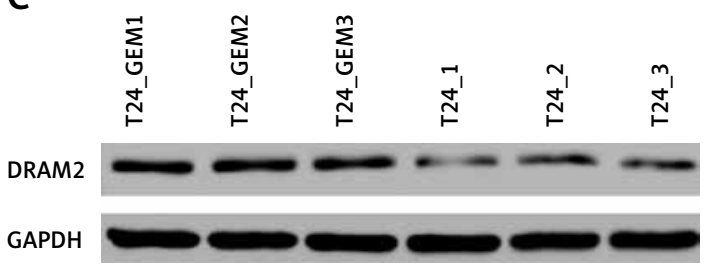

Figure 1. DRAM2 was up-regulated in gemcitabine-resistant cells. $\mathbf{A}$ - The heat map showed that DRAM2 was up-regulated in gemcitabine-resistant cells. B - The IC50 of T24-GEM cells $(9.953 \mu \mathrm{g} / \mathrm{ml})$ was significantly higher than that of T24 cells $(2.366 \mu \mathrm{g} / \mathrm{ml})$, and the cell viability of T24-GEM cells was higher compared with T24 cells. $\mathrm{C}$ - The expression level of DRAM2 in T24-GEM cells was remarkably higher than that in T24 cells as examined by western blot. ${ }^{*} P<0.01$ compared with T24 cells 
A

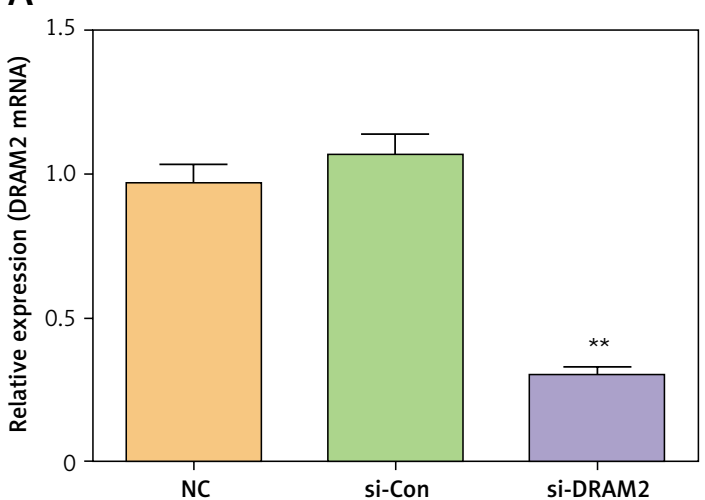

C

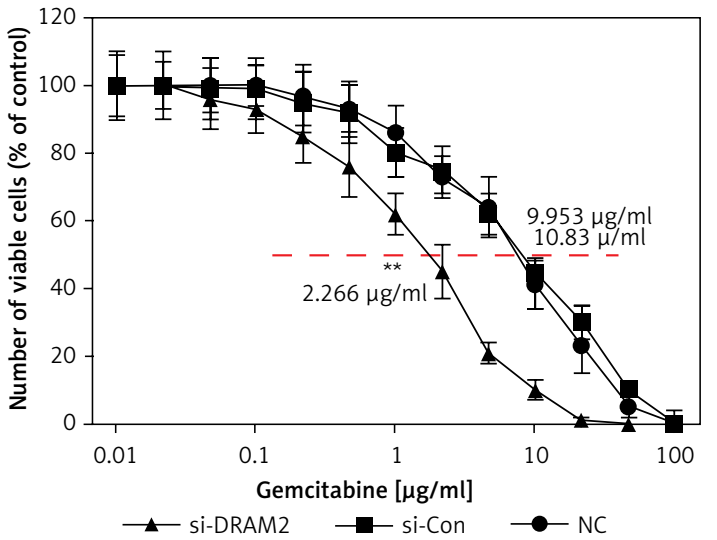

B

DRAM2

GAPDH

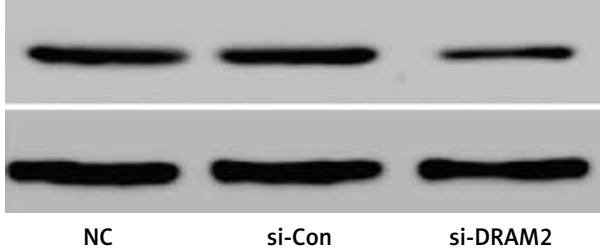

D

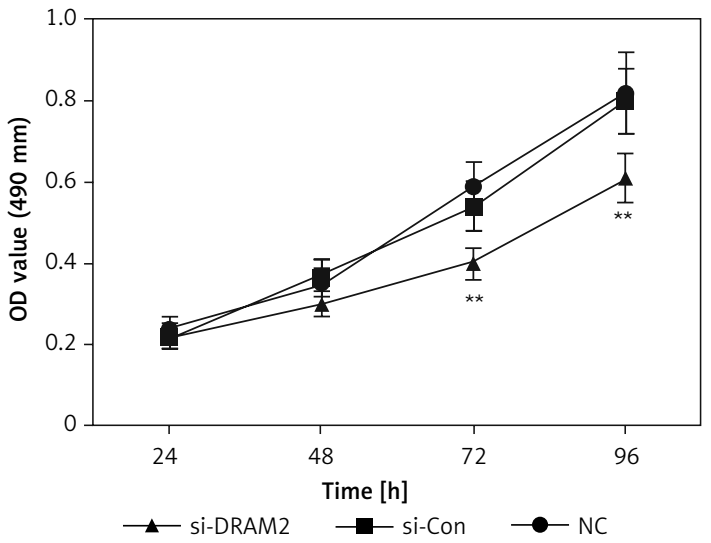

$\mathrm{E}$
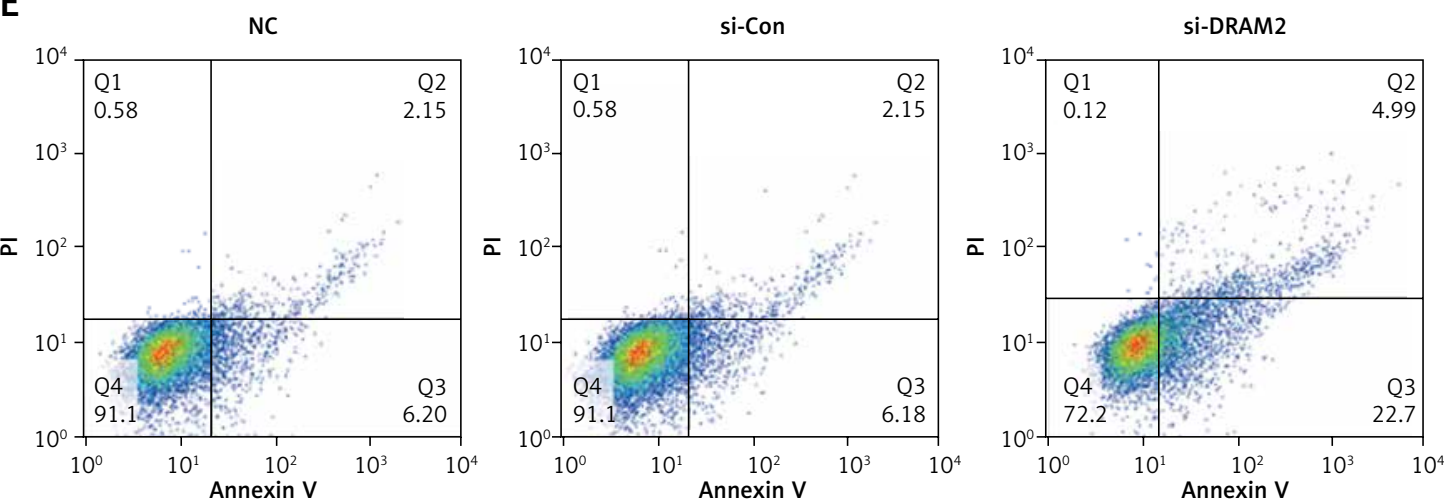

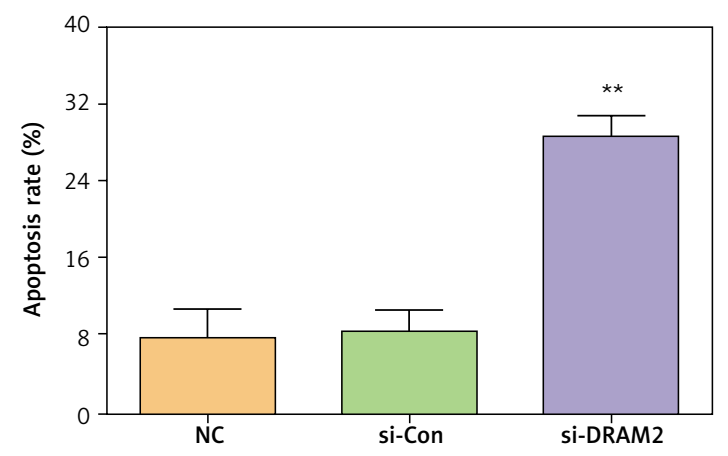

Figure 2. Knockdown of DRAM2 increased the sensitivity of T24-GEM cells to chemotherapy and inhibited autophagy. A - The expression of DRAM2 in T24-GEM cells significantly decreased after transfection with si-DRAM2 as measured by qRTPCR. B - The expression of DRAM2 in T24-GEM cells significantly decreased after transfection with siDRAM2 as measured by western blot. C - T24-GEM cells became more sensitive to gemcitabineinduced damage with the IC50 level decreasing from $9.953 \mu \mathrm{g} / \mathrm{ml}$ to $2.266 \mu \mathrm{g} / \mathrm{ml}$ after transfection with si-DRAM2. D - The cell growth of T24-GEM cells transfected with si-DRAM2 was suppressed significantly confirmed by MTT assay. E - The apoptosis rate of T24-GEM cells in si-DRAM2 groups sig nificantly increased in comparison with the si-Con group as detected by flow cytometry 
$\mathrm{F}$
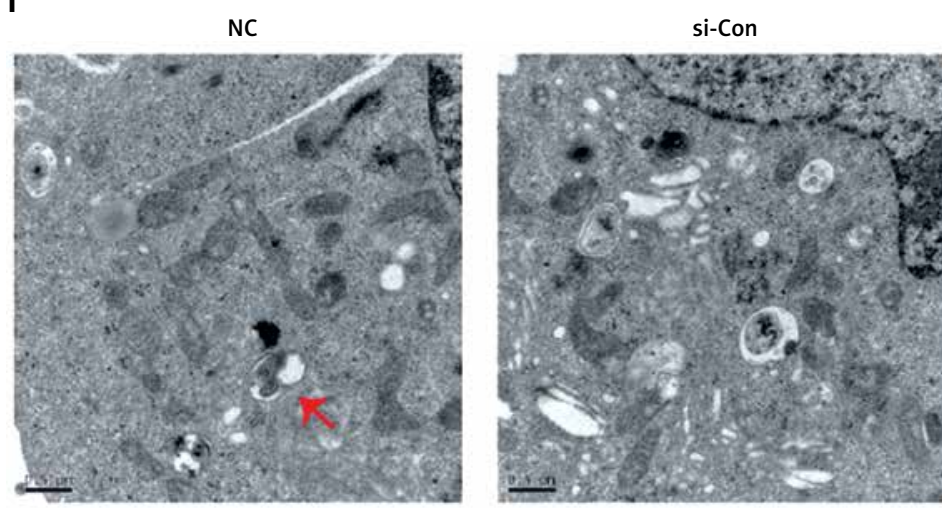

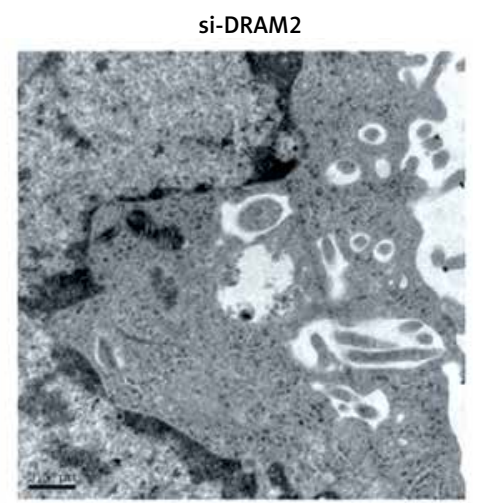

G

LC3-I
NC

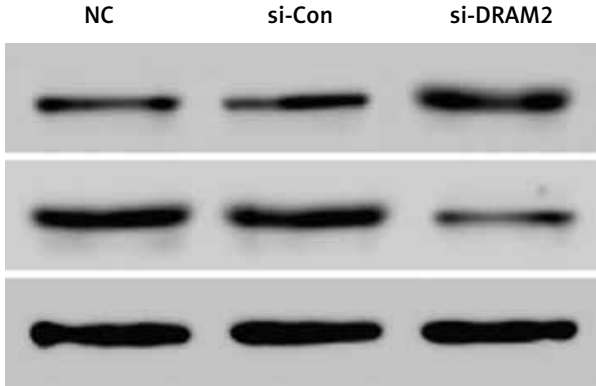

of DRAM2 increased the expression of $\mathrm{mRNA}$ and protein (Figures $3 \mathrm{~A}, \mathrm{~B}, p<0.01$ ). We also investigated the cell viability using MTT assays. The results showed that after over-expression of $D R A M 2$, the IC50 value increased to $3.595 \mu \mathrm{g} / \mathrm{ml}$, which was much higher than that in the pcDNA3.1 group $(1.672 \mu \mathrm{g} / \mathrm{ml}$, Figure $3 \mathrm{C}, p<0.01)$. These observations showed that the sensitivity to gemcitabine in T24 cells significantly decreased after over-expression of DRAM2. With the treatment of $0.5 \mu \mathrm{g} / \mathrm{mL}$ gemcitabine, cells transfected with DRAM2 were more active than cells in the pcDNA3.1 group (Figure $3 \mathrm{D}, p<0.01$ ), and the number of apoptotic cells decreased while autophagosomes increased (Figures $3 \mathrm{E}, \mathrm{F}, p<0.01$ ). Additionally, LC3 I expression level decreased while LC3 II expression level increased (Figure $3 \mathrm{G}$ ). These results suggested that over-expression of DRAM2 decreased the sensitivity of T24 cells to gemcitabine and promoted autophagy.

\section{Discussion}

Drug resistance is the major cause of the failure of clinical effectiveness of chemotherapy for bladder cancers. For example, cisplatin is one of the most effective bladder cancer agents, while inherent and acquired resistance limits its application [14]. The pyrimidine analog gemcitabine, alone or in combination with other drugs, is the current standard of care for advanced TCC. However, the response to gemcitabine in patients is
Figure 2. Cont. $\mathbf{F}$ - The number of autophagosome in T24-GEM cells in si-DRAM2 groups obviously decreased as shown by transmission electron microscopy. G - Autophagy markers LC3 I/II of T24-GEM cells in si-DRAM2 groups significantly increased as detected by western blot. NC - negative control group, T24-GEM cells were transfected with Lipofectamine 2000 reagent. Si-Con - siRNA control group, T24-GEM cells were transfected with SiRNA control sequence. si-DRAM2 - si-DRAM2 group, T24 cells were transfected with si-DRAM2 sequence. ${ }^{* \star} P<0.01$ compared with si-Con group

very poor, with no drastic reduction in metastasis or increase in the survival rate of patients [15]. Multiple molecular mechanisms of gemcitabine resistance encompassing different pathways have been suggested. Recently, Geller et al. hypothesized that intratumor bacteria might contribute to drug resistance of pancreatic ductal adenocarcinoma (PDAC) [16]. Amantini et al. demonstrated a mechanism of chemoresistance by which cancer cells increase intracellular cytidine pools that can in turn render gemcitabine ineffective by molecular competition [17]. Many studies have documented genetic aberrations in the development of BTCC, including point mutations of the TP53 [18], FHIT [19], KAI1 [20], BCL-2 and BCL-X genes [21]. Here, by screening the differentially expressed genes between T24 cells and T24-gemcitabine-resistant cells, DRAM2, DNA-damage regulated autophagy modulator 2, was found to be up-regulated in gemcitabine-resistant cells in our study.

DRAM2, a critical regulator of autophagy, encodes a transmembrane lysosomal protein thought to play a role in the initiation of autophagy [8]. It has been reported that DRAM2 expression is frequently dysregulated in cancers and that over-expression of DRAM2 induces autophagy [22]. DRAM2 was more highly expressed in gastric cancer (GC) tissues than in matched normal tissues [23]. Importantly, the knockdown phenotypes for DRAM2 inhibited autophagy and arrested myeloid cell differentiation in an acute promyelocytic leukemia (APL) mouse model [24]. 
A

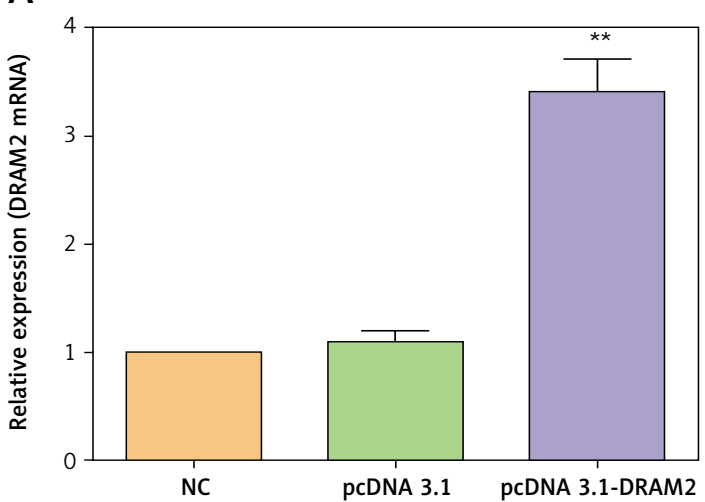

C

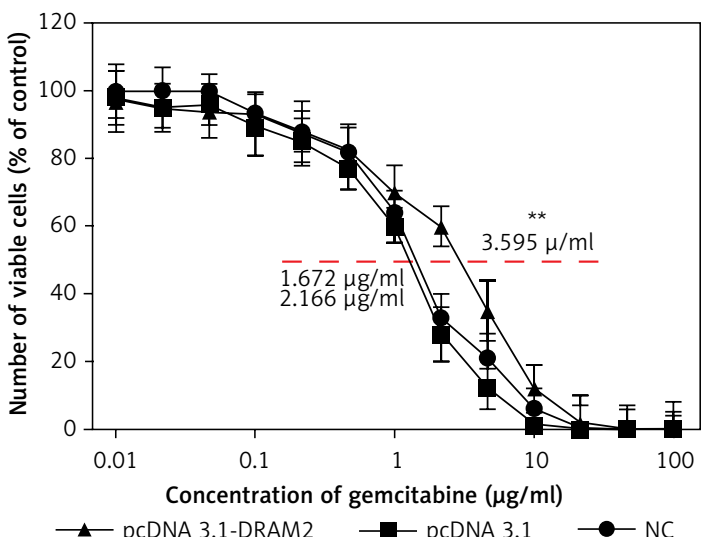

B

DRAM2
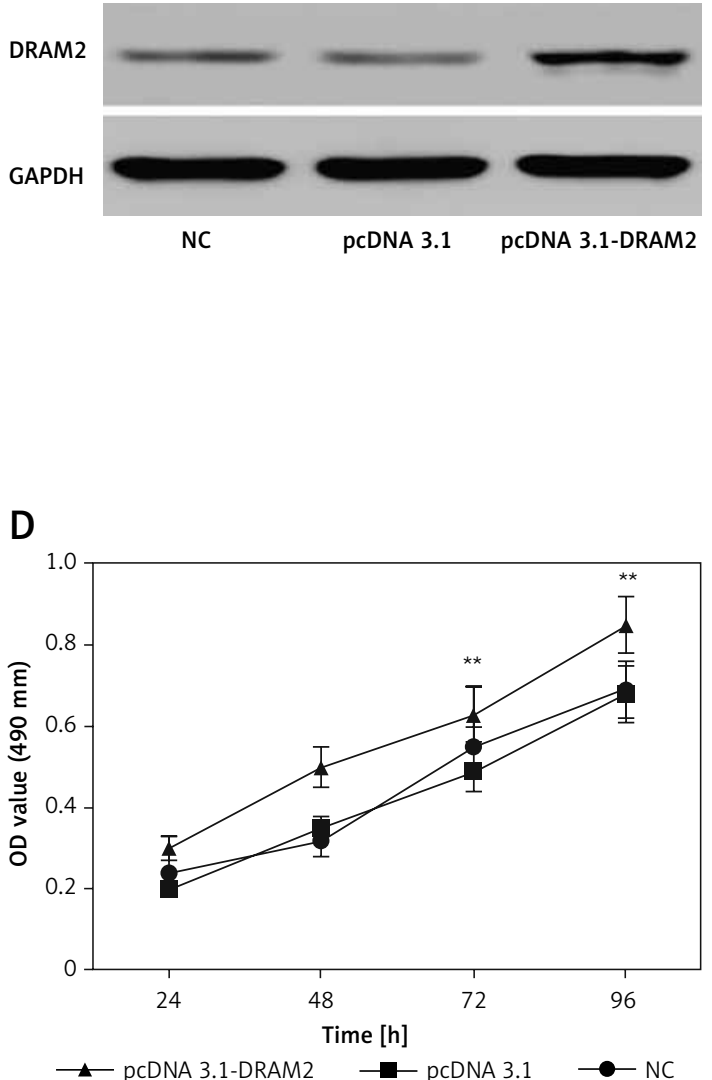

E
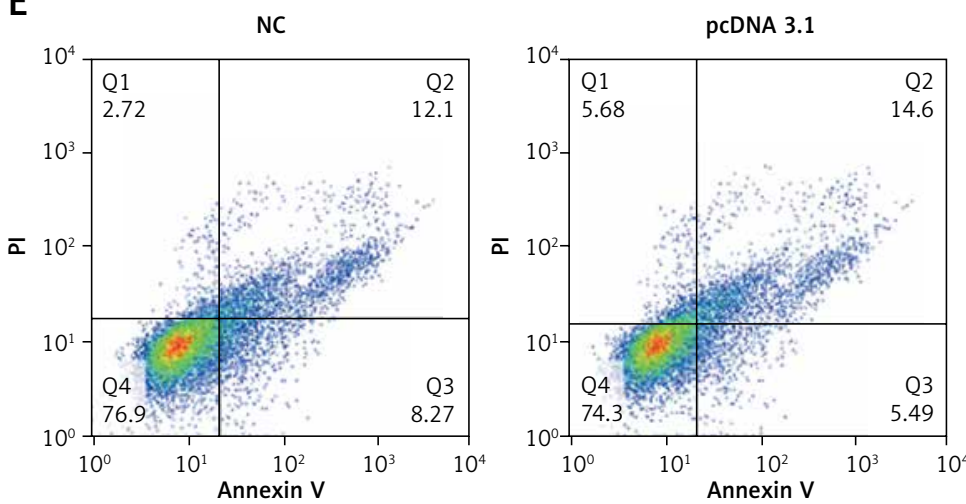

PCDNA 3.1-DRAM2

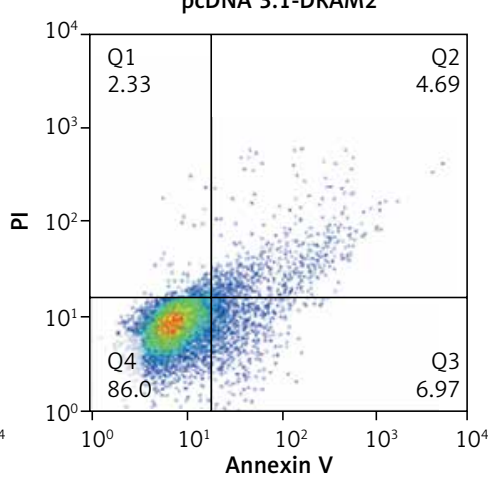

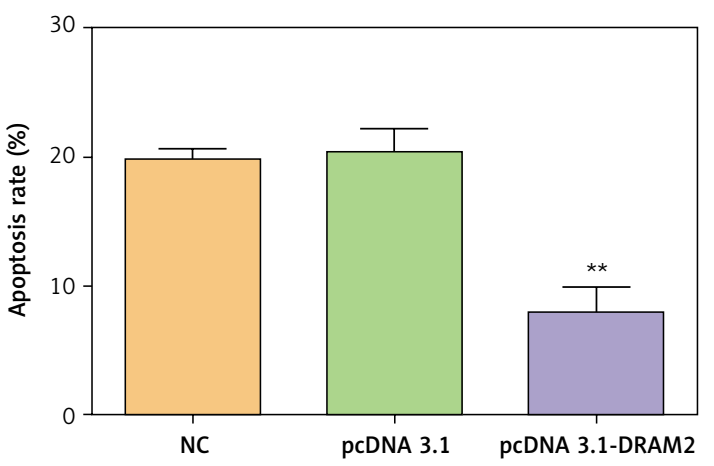

Figure 3. Over-expression of DRAM2 decreased the sensitivity of T24 cells to chemotherapy and promoted autophagy. $\mathbf{A}-$ The expression of DRAM 2 in T24 cells dramatically increased after transfection with pCDNA3.1-DRAM2 as observed by qRT-PCR. $\mathrm{B}$ - The expression of DRAM2 in pCDNA3.1 group of T24 cells was significantly higher than that in the pcDNA3.1 group as detected by western blot. C - After over-expression of DRAM2, the IC50 value increased to $3.595 \mu \mathrm{g} / \mathrm{ml}$, which was much higher than that in the pCDNA3.1 group. D - MTT assay showed that cell proliferation of T24 cells was significantly enhanced in the DRAM2 overexpression group compared with the pcDNA3.1 group. $\mathrm{E}$ - Compared with the pcDNA3.1 group, the apoptosis rate of T24 cells drastically increased after transfection with pcDNA3.1-DRAM2 as detected by flow cytometer 
$\mathrm{F}$

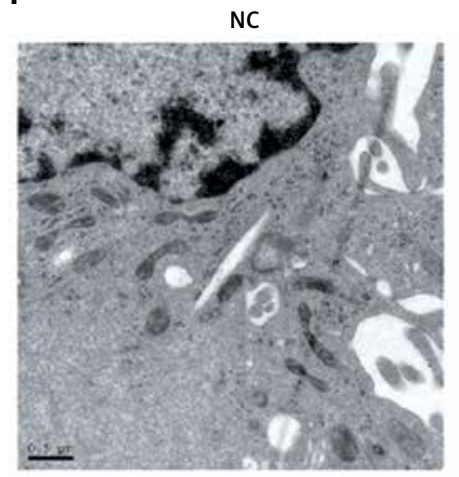

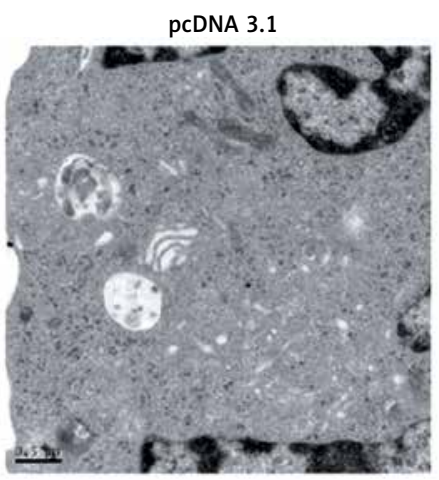

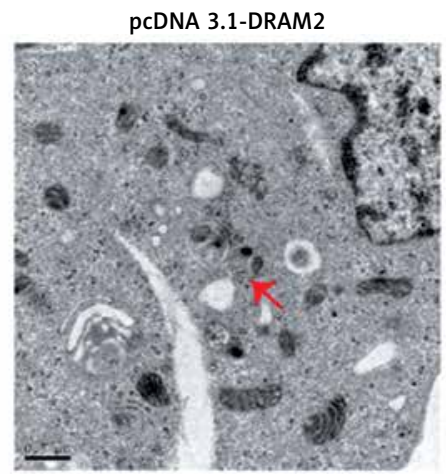

G

LC3-I

NC

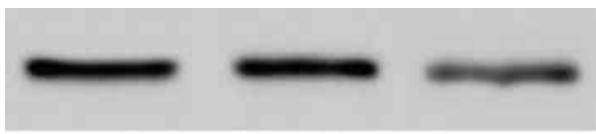

LC3-II

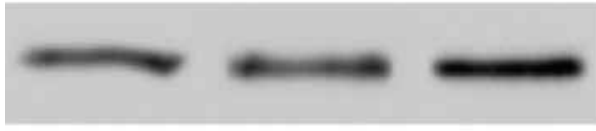

GAPDH

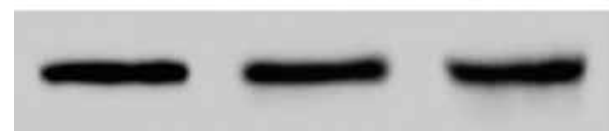

Figure 3. Cont. $\mathrm{F}-$ The number of autophagosome in T24 cells in the pcDNA3.1-DRAM2 group obviously increased as shown by transmission electron microscopy. G - Autophagy markers LC3 I/II of T24 cells in pcDNA3.1-DRAM2 groups significantly decreased as detected by western blot. NC - negative control group, T24 cells were transfected with the Lipofectamine 2000 reagent. pcDNA3.1 - pcDNA3.1 control group, T24 cells were transfected with empty pcDNA3.1 vector. pcDNA3.1-DRAM2 - pcDNA3.1-DRAM2 group, T24 cells were transfected with pcDNA3.1-DRAM2. ${ }^{* *} P<0.01$ compared with NC group
The DRAM2-dependent autophagic cascade affects Hodgkin lymphoma $(\mathrm{HL})$ cell proliferation and tumor growth [24]. Yoon et al. demonstrated that silencing of endogenous DRAM2 interferes with starvation-induced autophagy [22]. Similarly, our results showed that DRAM2 was up-regulated in T24-gemcitabine-resistant cells, which enhanced cell viability and inhibited cell apoptosis in T24-gemcitabine-resistant cells.

Dysregulated autophagy is highly prevalent in many types of cancer including drug-resistant bladder cancer and has been implicated in cytoprotection and tumor death [25-27]. Exploring the mechanism of gemcitabine resistance in bladder cancer from the perspective of autophagy may provide a brand new insight into solving the problem of drug resistance in bladder cancer. Previous studies have focused on suppressing autophagy-related genes or using autophagy inhibitors to strongly potentiate gemcitabine-induced apoptosis and inhibiting gemcitabine-induced autophagy [28]. Conflicts and controversies are often accompanied by studies of cancer cell autophagy. Some studies have indicated that in cancer autophagy exerts cytoprotective effects [29-31], whereas others suggest that autophagy positively contributes to cell death by enhancing cytotoxicity of anticancer drugs [32-34].

Our study firstly researched the dysregulated expression of the autophagy-related gene DRAM2 in T24 cells and T24-gemcitabine-resistant cells and explored resistance of gemcitabine altered through the regulation of DRAM2. Similar to studies of autophagy, studies on DRAM2 remain disputatious. In contrast to our results, Park et al. pointed out that expression of DRAM or DRAM 2 individually did not induce cell death, co-expression of DRAM2 with DRAM significantly induced cell death, while the silencing of endogenous DRAM2 attenuated cell death [35]. These conflicts may be caused by the stage and type of the cancer cells and the ignorance about drug-induced autophagy. Our study investigated the effects of DRAM2 on gemcitabine-resistant bladder cell biology. However, some limitations still exist. For instance, we only examined DRAM2 functions, while other genes and factors are also worth studying, such as micronutrients [36]. Furthermore, the pathway of DRAM2 in gemcitabine-resistant bladder cells needs to be clarified by future work.

In conclusion, overall, the current study demonstrated that DRAM2 was up-regulated in gemcitabine-resistant bladder cancer T24 cells. DRAM2 silence in T24-GEM cells promoted apoptosis whereas it inhibited autophagy and decreased the cell viability and resistance to gemcitabine, while in T24 cells, overexpression of DRAM2 promoted autophagy and cell viability but inhibited cell apoptosis and sensitivity to gemcitabine. These results allowed us to speculate that up-regulation of DRAM 2 may confer on gemcitabine-resistant bladder cancer cells an advantage of insensitivity to gemcitabine. Therefore, 
the treatment of bladder cancer with gemcitabine in combination with drugs targeting the autophagic gene DRAM 2 could represent an interesting new approach in bladder cancer therapy.

\section{Acknowledgments}

Baihetiya Azhati and Naibijiang Maolakuerban are first co-authors.

This study was supported by Natural Science Foundation of China (No. 81160088).

\section{Conflict of interest}

The authors declare no conflict of interest.

\section{References}

1. Yeung C, Dinh T, Lee J. The health economics of bladder cancer: an updated review of the published literature. Pharmacoeconomics 2014; 32: 1093-104.

2. Wang D, Feng JF, Yuan GY, Yang YH, Liu YS, Yang YW. Association between chromosomal aberration of exfoliated bladder cells in the urine and oxidative stress in patients with bladder transitional cell carcinoma. Oncol Lett 2017; 14: 137-44.

3. Wang ZP, Chen SY, Tian Y. Wild-type p53-induced phosphatase 1 is a prognostic marker and therapeutic target in bladder transitional cell carcinoma. Oncol Lett 2017; 13: 875-80.

4. Tao H, Chen F, Liu H, Hu Y, Wang Y, Li H. Wnt/betacatenin signaling pathway activation reverses gemcitabine resistance by attenuating Beclin1-mediated autophagy in the MG63 human osteosarcoma cell line. Mol Med Rep 2017; 16: 1701-6.

5. Kus T, Aktas G. Maintenance treatment with gemcitabine have a promising activity on metastatic bladder cancer survival. Turk J Urol 2017; 43: 273-8.

6. Aydinlik S, Erkisa M, Cevatemre B, et al. Enhanced cytotoxic activity of doxorubicin through the inhibition of autophagy in triple negative breast cancer cell line. Biochim Biophys Acta 2017; 1861: 49-57.

7. Tan Q, Wang H, Hu Y, et al. Src/STAT3-dependent heme oxygenase-1 induction mediates chemoresistance of breast cancer cells to doxorubicin by promoting autophagy. Cancer Sci 2015; 106: 1023-32.

8. El-Asrag ME, Sergouniotis PI, McKibbin M, et al. Biallelic mutations in the autophagy regulator DRAM2 cause retinal dystrophy with early macular involvement. Am J Hum Genet 2015; 96: 948-54.

9. Bai S, Tian B, Li A, Yao Q, Zhang G, Li F. MicroRNA-125b promotes tumor growth and suppresses apoptosis by targeting DRAM2 in retinoblastoma. Eye (Lond) 2016; 30: 1630-8.

10. Sun XY, Qu Y, Ni AR, et al. Novel histone deacetylase inhibitor N25 exerts anti-tumor effects and induces autophagy in human glioma cells by inhibiting HDAC3. Oncotarget 2017; 8: 75232-42.

11. Huang XL, Zhang $\mathrm{H}$, Yang $\mathrm{XY}$, et al. Activation of a c-Jun $\mathrm{N}$-terminal kinase-mediated autophagy pathway attenuates the anticancer activity of gemcitabine in human bladder cancer cells. Anticancer Drugs 2017; 28: 596602.

12. Peng Y, Cao J, Yao XY, et al. TUSC3 induces autophagy in human non-small cell lung cancer cells through Wnt/ beta-catenin signaling. Oncotarget 2017; 8: 52960-74.
13. Huang YH, Sun Y, Huang FY, et al. Toxicarioside $O$ induces protective autophagy in a sirtuin-1-dependent manner in colorectal cancer cells. Oncotarget 2017; 8: 52783-91.

14. Monneret C. Platinum anticancer drugs. From serendipity to rational design. Ann Pharm Fr 2011; 69: 286-95.

15. Heinemann V, Wilke H, Mergenthaler HG, et al. Gemcitabine and cisplatin in the treatment of advanced or metastatic pancreatic cancer. Ann Oncol 2000; 11: 1399-403.

16. Geller LT, Barzily-Rokni M, Danino T, et al. Potential role of intratumor bacteria in mediating tumor resistance to the chemotherapeutic drug gemcitabine. Science 2017; 357: 1156-60.

17. Amantini C, Morelli MB, Nabissi M, et al. Capsaicin triggers autophagic cell survival which drives epithelial mesenchymal transition and chemoresistance in bladder cancer cells in an Hedgehog-dependent manner. Oncotarget 2016; 7: 50180-94.

18. Olsson H, Hultman P, Rosell J, Soderkvist P, Jahnson S. MDM2 SNP309 promoter polymorphism and p53 mutations in urinary bladder carcinoma stage T1. BMC Urol 2013; 13: 5.

19. Skopelitou AS, Gloustianou G, Bai M, Huebner K. FHIT gene expression in human urinary bladder transitional cell carcinomas. In Vivo 2001; 15: 169-73.

20. Ow K, Delprado W, Fisher R, et al. Relationship between expression of the KAl1 metastasis suppressor and other markers of advanced bladder cancer. J Pathol 2000; 191: 39-47.

21. Kirsh EJ, Baunoch DA, Stadler WM. Expression of bcl-2 and bcl-X in bladder cancer. J Urol 1998; 159: 1348-53.

22. Yoon JH, Her S, Kim M, Jang IS, Park J. The expression of damage-regulated autophagy modulator 2 (DRAM2) contributes to autophagy induction. Mol Biol Rep 2012; 39: 1087-93.

23. Anami K, Oue N, Noguchi T, et al. Search for transmembrane protein in gastric cancer by the Escherichia coli ampicillin secretion trap: expression of DSC2 in gastric cancer with intestinal phenotype. J Pathol 2010; 221: 275-84.

24. Gillet P, Bannwarth B, Chambre JF, Pere P, Gaucher A. Enterocolitis induced by gold salts. The value of sodium cromoglycate. Therapie 1989; 44: 305.

25. Konstantakou EG, Voutsinas GE, Velentzas AD, et al. 3-BrPA eliminates human bladder cancer cells with highly oncogenic signatures via engagement of specific death programs and perturbation of multiple signaling and metabolic determinants. Mol Cancer 2015; 14: 135.

26. Ojha R, Singh SK, Bhattacharyya $S$, et al. Inhibition of grade dependent autophagy in urothelial carcinoma increases cell death under nutritional limiting condition and potentiates the cytotoxicity of chemotherapeutic agent. J Urol 2014; 191: 1889-98.

27. Yang MC, Wang HC, Hou YC, Tung HL, Chiu TJ, Shan YS. Blockade of autophagy reduces pancreatic cancer stem cell activity and potentiates the tumoricidal effect of gemcitabine. Mol Cancer 2015; 14: 179.

28. Huang YT, Cheng CC, Lin TC, Chiu TH, Lai PC. Therapeutic potential of sepantronium bromide YM155 in gemcitabine-resistant human urothelial carcinoma cells. Oncol Rep 2014; 31: 771-80.

29. Murrow L, Debnath J. Autophagy as a stress-response and quality-control mechanism: implications for cell injury and human disease. Annu Rev Pathol 2013; 8: 105-37.

30. Lee JS, Li Q, Lee JY, et al. FLIP-mediated autophagy regulation in cell death control. Nat Cell Biol 2009; 11: 1355-62. 
31. Rubinsztein DC, Gestwicki JE, Murphy LO, Klionsky DJ. Potential therapeutic applications of autophagy. Nat Rev Drug Discov 2007; 6: 304-12.

32. Fullgrabe J, Lynch-Day MA, Heldring N, et al. The histone $\mathrm{H} 4$ lysine 16 acetyltransferase hMOF regulates the outcome of autophagy. Nature 2013; 500: 468-71.

33. Weiner LM, Lotze MT. Tumor-cell death, autophagy, and immunity. N Engl J Med 2012; 366: 1156-8.

34. McPhee CK, Logan MA, Freeman MR, Baehrecke EH. Activation of autophagy during cell death requires the engulfment receptor Draper. Nature 2010; 465: 1093-6.

35. Park SM, Kim K, Lee EJ, et al. Reduced expression of DRAM2/TMEM77 in tumor cells interferes with cell death. Biochem Biophys Res Commun 2009; 390: 1340-4.

36. Golabek T, Bukowczan J, Sobczynski R, Leszczyszyn J, Chlosta PL. The role of micronutrients in the risk of urinary tract cancer. Arch Med Sci 2016; 12: 436-47. 